\title{
FINDING LINKING SETS
}

\author{
MARTIN SCHECHTER AND KYRIL TINTAREV
}

Received 10 May 2006; Accepted 11 May 2006

We present the most general definition of the linking of sets in a Banach space and discuss necessary and sufficient conditions for sets to link.

Copyright (c) 2006 Hindawi Publishing Corporation. All rights reserved.

\section{Introduction}

Many nonlinear problems in the physical and social sciences can be reduced to finding critical points (minima, maxima, and minimax points) of functionals (real-valued functions on various spaces). The first critical points to be studied were maxima and minima, and much of the activity in the calculus of variations has been devoted to the finding of such points. A more difficult problem is to find critical points that are neither maxima nor minima.

It was discovered that there exist sets having the property that critical points can be found when the sets separate a functional. This has led to the search for such sets. Specifically, it is desired to find sets with the following properties.

Definition 1.1. Let $A, B$ be subsets of a Banach space $E$. Say that $A$ links $B$ if the class of $G \in C_{U}^{1}(E ; \mathbb{R})$ satisfying

$$
a_{0}:=\sup _{A} G<b_{0}:=\inf _{B} G
$$

is nonempty, and for every such $G$, there are a sequence $\left\{u_{k}\right\} \subset E$ and a constant $c$ such that

$$
\begin{gathered}
b_{0} \leq c<\infty, \\
G\left(u_{k}\right) \longrightarrow c, \quad G^{\prime}\left(u_{k}\right) \longrightarrow 0 .
\end{gathered}
$$

(In this definition, as well as in the rest of the paper, we consider functionals of the class $C_{U}^{m} \subset C^{m}$, i.e., functionals whose Fréchet derivatives up to the order $m$ are uniformly continuous on bounded sets.) 
The importance of the concept of linking stems from the fact that in many applications, a sequence satisfying (1.3) (called a Palais-Smale sequence) leads to a critical point of $G$. In particular, this is true if any sequence satisfying (1.3) has a convergent subsequence. (If this is the case, then $G$ is said to satisfy the Palais-Smale condition.) Thus, a useful method of finding critical points of $G$ is to find two subsets $A, B$ of $E$ such that $A$ links $B$ and (1.3) holds.

There are several sufficient conditions given in the literature which imply that a set $A$ links a set $B$ in this sense (cf., e.g., $[1,3,5-8,11,14,15])$. The most comprehensive is that of [11].

In [11], our goal was to prove a theorem of the following form.

Theorem 1.2. Let $G$ be a $C^{1}$-functional on a Banach space $E$, and let $A, B$ be subsets of $E$ such that $A$ has a certain relationship to $B$. Assume that

$$
a_{0}:=\sup _{A} G<b_{0}:=\inf _{B} G
$$

Then there are a sequence $\left\{u_{k}\right\} \subset E$ and an $a \geq b_{0}$ such that

$$
G\left(u_{k}\right) \longrightarrow a, \quad G^{\prime}\left(u_{k}\right) \longrightarrow 0
$$

The reason for the requirement that $A$ have a relationship to $B$ is that the theorem is obviously false if $A$ and $B$ are two arbitrary sets. The problem facing us was to find a very general relationship that would make the theorem true. We defined a relationship which indeed made the theorem true and was the most general known hypothesis that did so. We termed this relationship "linking." (Previous authors used this term to describe more restrictive definitions.) We suspect that our definition of linking is the most general possible definition.

In the present paper, we define two relationships which are close to each other. The stronger one is sufficient for linking while the weaker one is necessary. We use the following maps.

Definition 1.3. Say that a map $\varphi: E \rightarrow E$ is of class $\Lambda$, if it is a homeomorphism onto $E$, and both $\varphi, \varphi^{-1}$ are bounded on bounded sets. If, furthermore, $\varphi, \varphi^{-1} \in C_{U}^{1}(E ; E)$, say that $\varphi \in \Lambda_{U}$.

Definition 1.4. Say that a bounded set $A$ is chained to a set $B$ if $A \cap B=\phi$ and

$$
\inf _{x \in B}\|\varphi(x)\| \leq \sup _{x \in A}\|\varphi(x)\|, \quad \varphi \in \Lambda_{U}
$$

Definition 1.5. Say that a bounded $A$ is strongly chained to $B$ if $A \cap B=\phi$ and (1.6) holds for every $\varphi \in \Lambda$.

Definition 1.6. For $A \subset E$, define

$$
\mathscr{K}_{U}(A)=\left\{\varphi^{-1}\left(B_{R}\right): \varphi \in \Lambda_{U}, \varphi(A) \subset B_{R}\right\},
$$


where

$$
B_{R}=\{u \in E:\|u\|<R\}
$$

Our main results are the following.

Theorem 1.7. If a bounded set $A$ is strongly chained to $B$, then $A$ links $B$.

Theorem 1.8. Let $G$ be a $\left(C^{2} \cap C_{U}\right)$-functional on $E$, and let $A, B$ be nonempty subsets of E such that $A$ is bounded and chained to $B$ and

$$
a_{0}:=\sup _{A} G \leq b_{0}:=\inf _{B} G
$$

Let

$$
a:=\inf _{K \in \mathscr{K}_{U}(A)} \sup _{K} G
$$

Then there is a sequence $\left\{u_{k}\right\} \subset E$ such that

$$
G\left(u_{k}\right) \longrightarrow a, \quad G^{\prime}\left(u_{k}\right) \longrightarrow 0
$$

If $a=b_{0}$, then it can be also required

$$
d\left(u_{k}, B\right) \longrightarrow 0
$$

Theorem 1.9. If $E$ is a Hilbert space, and $A$ links $B$, then it is chained to $B$.

Theorem 1.9 with additional assumptions is due to the authors elsewhere [12]. There it was proved that in a Hilbert space a compact set links another set if and only if it is chained to it.

\section{The strong case}

Definition 2.1. For $A \subset E$, define

Lemma 2.2. One has

$$
\begin{gathered}
\Lambda(A)=\{\varphi \in \Lambda: \varphi(u)=u, u \in A\}, \\
\Lambda_{U}(A)=\left\{\varphi \in \Lambda_{U}: \varphi(u)=u, u \in A\right\} .
\end{gathered}
$$

$$
\begin{aligned}
\varphi_{1}, \varphi_{2} \in \Lambda & \Longrightarrow \varphi_{1} \circ \varphi_{2} \in \Lambda, \\
\varphi_{1}, \varphi_{2} \in \Lambda_{U} & \Longrightarrow \varphi_{1} \circ \varphi_{2} \in \Lambda_{U}, \\
\varphi_{1}, \varphi_{2} \in \Lambda(A) & \Longrightarrow \varphi_{1} \circ \varphi_{2} \in \Lambda(A), \\
\varphi_{1}, \varphi_{2} \in \Lambda_{U}(A) & \Longrightarrow \varphi_{1} \circ \varphi_{2} \in \Lambda_{U}(A) .
\end{aligned}
$$


$4 \quad$ Finding linking sets

Lemma 2.3. One has

$$
\begin{aligned}
\varphi \in \Lambda & \text { iff } \varphi^{-1} \in \Lambda, \\
\varphi \in \Lambda_{U} & \text { iff } \varphi^{-1} \in \Lambda_{U}, \\
\varphi \in \Lambda(A) & \text { iff } \varphi^{-1} \in \Lambda(A), \\
\varphi \in \Lambda_{U}(A) & \text { iff } \varphi^{-1} \in \Lambda_{U}(A) .
\end{aligned}
$$

Definition 2.4. For $A \subset E$, define

$$
\mathscr{K}(A)=\left\{\varphi^{-1}\left(B_{R}\right): \varphi \in \Lambda, \varphi(A) \subset B_{R}\right\} .
$$

Lemma 2.5. If $A$ is strongly chained to $B$ and $K \in \mathscr{K}(A)$, then $K \cap B \neq \phi$.

Proof. There is a $\varphi \in \Lambda$ such that $K=\varphi^{-1}\left(B_{R}\right)$ with $\varphi(A) \subset B_{R}$. Since $A$ is strongly chained to $B$, we have

$$
\inf _{x \in B}\|\varphi(x)\| \leq \sup _{x \in A}\|\varphi(x)\|<R
$$

Hence, there is a $v \in B$ such that $\varphi(v) \in B_{R}$. Hence, $v=\varphi^{-1} \varphi(v) \in \varphi^{-1}\left(B_{R}\right)$.

Lemma 2.6. If $K \in \mathscr{K}(A)$ and $\sigma \in \Lambda(A)$, then $\sigma(K) \in \mathscr{K}(A)$.

Proof. There is a $\varphi \in \Lambda$ such that $K=\varphi^{-1}\left(B_{R}\right)$ with $\varphi(A) \subset B_{R}$. Let

$$
\tilde{\varphi}=\varphi \circ \sigma^{-1}
$$

Then $\tilde{\varphi} \in \Lambda$ (Lemma 2.2). Now,

$$
\widetilde{\varphi}^{-1}=\sigma \circ \varphi^{-1}
$$

Hence,

$$
\sigma(K)=\sigma\left[\varphi^{-1}\left(B_{R}\right)\right]=\tilde{\varphi}^{-1}\left(B_{R}\right) .
$$

Moreover,

$$
\tilde{\varphi}(u)=\varphi(u), \quad u \in A
$$

Hence,

$$
\sup _{x \in A}\|\tilde{\varphi}(x)\|=\sup _{x \in A}\|\varphi(x)\|<R .
$$

Therefore, $\sigma(K) \in \mathscr{K}(A)$.

We can now give the proof of Theorem 1.7. 
Proof. Let $G$ be a $\left(C^{1} \cap C_{U}\right)$-functional on $E$, and let $A, B$ be nonempty subsets of $E$ such that $A$ is strongly chained to $B$ and (1.9) holds. Then

$$
a:=\inf _{K \in \mathscr{K}(A)} \sup _{K} G
$$

is finite. By Lemma 2.5, $K \cap B \neq \phi$ for all $K \in \mathscr{K}(A)$. Hence, $a \geq b_{0}$. Assume first that $b_{0}<a$. If (1.11) were false, there would exist a positive constant $\delta$ such that $3 \delta<a-b_{0}$ and

$$
\left\|G^{\prime}(u)\right\| \geq 3 \delta
$$

whenever

$$
u \in Q=\{u \in E:|G(u)-a| \leq 3 \delta\}
$$

Since $G \in C^{1}(E, \mathbb{R})$, there is a locally Lipschitz continuous mapping $Y(u)$ of $\widehat{E}=\{u \in E$ : $G^{\prime}(u) \neq 0$ into $E$ such that

$$
\begin{gathered}
\|Y(u)\| \leq 1, \quad u \in \hat{E} \\
\left(G^{\prime}(u), Y(u)\right) \geq 2 \delta
\end{gathered}
$$

whenever $u$ satisfies (2.13). Let

$$
\begin{gathered}
Q_{0}=\{u \in E:|G(u)-a| \leq 2 \delta\}, \\
Q_{1}=\{u \in E:|G(u)-a| \leq \delta\} \\
Q_{2}=E \backslash Q_{0} \\
\eta(u)=\frac{d\left(u, Q_{2}\right)}{\left[d\left(u, Q_{1}\right)+d\left(u, Q_{2}\right)\right]} .
\end{gathered}
$$

It is easily checked that $\eta(u)$ is locally Lipschitz continuous on $E$ and satisfies

$$
\eta(u)=1, \quad u \in Q_{1} ; \quad \eta(u)=0, \quad u \in \bar{Q}_{2} ; \quad 0<\eta(u)<1, \quad \text { otherwise. }
$$

Consider the differential equation

$$
\sigma^{\prime}(t)=W(\sigma(t)), \quad t \in \mathbb{R}, \sigma(0)=u,
$$

where

$$
W(u)=-\eta(u) Y(u)
$$

The mapping $W$ is locally Lipschitz continuous on the whole of $E$ and is bounded in norm by 1 . Hence by a well-known existence theorem for ordinary differential equations in a Banach space, (2.18) has a unique solution for all $t \in \mathbb{R}$. Let us denote the solution 
$6 \quad$ Finding linking sets

of (2.18) by $\sigma(t) u$. The mapping $\sigma(t)$ is in $C(E \times \mathbb{R}, E)$ and is called the flow generated by (2.19). Note that

$$
\begin{aligned}
\frac{d G(\sigma(t) u)}{d t} & =\left(G^{\prime}(\sigma(t) u), \sigma^{\prime}(t) u\right) \\
& =-\eta(\sigma(t) u)\left(G^{\prime}(\sigma(t) u), Y(\sigma(t) u)\right) \\
& \leq-2 \delta \eta(\sigma(t) u) .
\end{aligned}
$$

Thus,

$$
\begin{gathered}
G(\sigma(t) u) \leq G(u), \quad t \geq 0, \\
G(\sigma(t) u) \leq a_{0}, \quad u \in A, t \geq 0, \\
\sigma(t) u=u, \quad u \in A, t \geq 0 .
\end{gathered}
$$

This follows from the fact that

$$
G(\sigma(t) u) \leq a_{0} \leq b_{0}<a-3 \delta, \quad u \in A, t \geq 0 .
$$

Hence, $\eta(\sigma(t) u)=0$ for $u \in A, t \geq 0$. This means that

$$
\begin{gathered}
\sigma^{\prime}(t) u=0, \quad u \in A, t \geq 0, \\
\sigma(t) u=\sigma(0) u=u, \quad u \in A, t \geq 0 .
\end{gathered}
$$

Let

$$
E_{\alpha}=\{u \in E: G(u) \leq \alpha\}
$$

There is a $T>0$ such that

$$
\sigma(T) E_{a+\delta} \subset E_{a-\delta}
$$

In fact, we can take $T=1$. Let $u$ be any element in $E_{a+\delta}$. If there is a $t_{1} \in[0, T]$ such that $\sigma\left(t_{1}\right) u \notin Q_{1}$, then

$$
G(\sigma(T) u) \leq G\left(\sigma\left(t_{1}\right) u\right)<a-\delta
$$

by (2.20). Hence $\sigma(T) u \in E_{a-\delta}$. On the other hand, if $\sigma(t) u \in Q_{1}$ for all $t \in[0, T]$, then $\eta(\sigma(t) u)=1$ for all $t$, and (2.20) yields

$$
G(\sigma(T) u) \leq G(u)-2 \delta T \leq a-\delta .
$$

Hence (2.25) holds. Now by (2.11), there is a $K \in \mathscr{K}(A)$ such that

$$
K \subset E_{a+\delta}
$$


Note that $\sigma(T) \in \Lambda(A)$. Let $\tilde{K}=\sigma(T)(K)$. Then $\tilde{K} \in \mathscr{K}(A)$ by Lemma 2.6. But

$$
\sup _{\widetilde{K}} G=\sup _{u \in K} G(\sigma(T) u)<a-\delta
$$

which contradicts (2.11), proving the theorem for the case $b_{0}<a$.

Now assume $b_{0}=a$. If there did not exist a sequence satisfying both (1.11)-(1.12), then there would be positive numbers $\epsilon, \delta, T$ such that $\delta<\epsilon T$ and (2.12) holds whenever

$$
u \in Q=\{u \in E: d(u, B) \leq 4 T,|G(u)-a| \leq 3 \delta\} .
$$

Let

$$
\begin{aligned}
& Q_{0}=\{u \in E: d(u, B) \leq 3 T,|G(u)-a| \leq 2 \delta\}, \\
& Q_{1}=\{u \in E: d(u, B) \leq 2 T,|G(u)-a| \leq \delta\} .
\end{aligned}
$$

Since $a=b_{0}$, we see that $Q_{1} \neq \phi$. Define $Q_{2}$ and $\eta(u)$ as before and let $\sigma(t)$ be the flow generated by the mapping (2.19) with everything now with respect to the new sets $Q_{j}$. Let $u$ be any element in $E_{a+\delta}$. If there is a $t_{1} \leq T$ such that $\sigma\left(t_{1}\right) u \notin Q_{1}$, then either

$$
G\left(\sigma\left(t_{1}\right) u\right)<a-\delta
$$

or

$$
d\left(\sigma\left(t_{1}\right) u, B\right)>2 T \text {. }
$$

Since

$$
\left\|\sigma(t) u-\sigma\left(t^{\prime}\right) u\right\| \leq\left|t-t^{\prime}\right|
$$

by (2.14), (2.33) implies that

$$
d(\sigma(t) u, B)>T, \quad 0 \leq t \leq T .
$$

On the other hand, if $\sigma(t) u \in Q_{1}$ for all $t \in[0, T]$, then

$$
G(\sigma(T) u) \leq G(u)-2 \epsilon T \leq a+\delta-2 \delta=a-\delta .
$$

Thus we have either

$$
G(\sigma(T) u)<a-\delta
$$

or (2.35) holds. Since $b_{0}=a$, this shows that

$$
\sigma(T) E_{a+\delta} \cap B=\phi .
$$

We also note that

$$
\sigma(t) A \cap B=\phi, \quad 0 \leq t \leq T .
$$


We have by (2.20) that

$$
G(\sigma(t) u) \leq a_{0}-2 \epsilon \int_{0}^{t} \eta(\sigma(\tau) u) d \tau, \quad u \in A
$$

If $\sigma(t) u \in B$, we must have $G(\sigma(t) u) \geq b_{0} \geq a_{0}$. The only way this can happen is when

$$
\eta(\sigma(\tau) u) \equiv 0, \quad 0 \leq \tau \leq t
$$

But this implies $\sigma(\tau) u \in \bar{Q}_{2}$ for such $\tau$, and this in turn implies either

$$
G(\sigma(\tau) u)<a-\delta, \quad 0 \leq \tau \leq t
$$

or

$$
d(\sigma(\tau) u, B)>2 T, \quad 0 \leq \tau \leq t
$$

In either case, we cannot have $\sigma(t) u \in B$. Thus (2.39) holds. Let $K \in \mathscr{K}(A)$ satisfy (2.28), and let $\tilde{K}=\sigma(T) K$. Then $\tilde{K} \in \mathscr{K}(A)$. But (2.38) and (2.39) imply that $\widetilde{K} \cap B=\phi$, contradicting the fact that $A$ is strongly chained to $B$. This completes the proof of the theorem.

\section{The remaining proofs}

Lemma 3.1. If $A$ is chained to $B$ and $K \in \mathscr{K}_{U}(A)$, then $K \cap B \neq \phi$.

Proof. There is a $\varphi \in \Lambda_{U}$ such that $K=\varphi^{-1}\left(B_{R}\right)$ with $\varphi(A) \subset B_{R}$. Since $A$ is chained to $B$, we have

$$
\inf _{x \in B}\|\varphi(x)\| \leq \sup _{x \in A}\|\varphi(x)\|<R
$$

Hence, there is a $v \in B$ such that $\varphi(v) \in B_{R}$. Hence, $v=\varphi^{-1} \varphi(v) \in \varphi^{-1}\left(B_{R}\right)$.

Lemma 3.2. If $K \in \mathscr{K}_{U}(A)$ and $\sigma \in \Lambda_{U}(A)$, then $\sigma(K) \in \mathscr{K}_{U}(A)$.

We can now give the proof of Theorem 1.8.

Proof. Let $G$ be a $\left(C^{2} \cap C_{U}\right)$-functional on $E$, and let $A, B$ be nonempty subsets of $E$ such that $A$ is chained to $B$ and (1.9) holds. Then $a$ given by (1.10) is finite. By Lemma 3.1, $K \cap B \neq \phi$ for all $K \in \mathscr{K}_{U}(A)$. Hence, $a \geq b_{0}$. Assume first that $b_{0}<a$. If (1.11) were false, there would exist a positive constant $\delta$ such that $3 \delta<a-b_{0}$ and

$$
\left\|G^{\prime}(u)\right\| \geq 3 \delta
$$

whenever

$$
u \in Q=\{u \in E:|G(u)-a| \leq 3 \delta\} .
$$


Let

$$
\begin{gathered}
Q_{0}=\{u \in E:|G(u)-a| \leq 2 \delta\}, \\
Q_{1}=\{u \in E:|G(u)-a| \leq \delta\}, \\
Q_{2}=E \backslash Q_{0}, \\
\eta(u)=\frac{d\left(u, Q_{2}\right)}{\left[d\left(u, Q_{1}\right)+d\left(u, Q_{2}\right)\right]} .
\end{gathered}
$$

It is easily checked that $\eta(u)$ is locally Lipschitz continuous on $E$ and satisfies

$$
\eta(u)=1, \quad u \in Q_{1} ; \quad \eta(u)=0, \quad u \in \bar{Q}_{2} ; \quad 0<\eta(u)<1, \quad \text { otherwise. }
$$

Consider the differential equation

$$
\sigma^{\prime}(t)=W(\sigma(t)), \quad t \in \mathbb{R}, \sigma(0)=u,
$$

where

$$
W(u)=\frac{-\eta(u) G^{\prime}(u)}{\left\|G^{\prime}(u)\right\|}
$$

Since $G \in C^{2}(E, \mathbb{R})$, the mapping $W$ is locally Lipschitz continuous on the whole of $E$ and is bounded in norm by 1 . Hence by a well-known existence theorem for ordinary differential equations in a Banach space, (3.6) has a unique solution for all $t \in \mathbb{R}$. Let us denote the solution of (3.6) by $\sigma(t) u$. The mapping $\sigma(t)$ is in $C_{U}^{1}(E \times \mathbb{R}, E)$ and is called the flow generated by (3.7). Note that

$$
\begin{aligned}
\frac{d G(\sigma(t) u)}{d t} & =\left(G^{\prime}(\sigma(t) u), \sigma^{\prime}(t) u\right) \\
& =-\eta(\sigma(t) u)\left\|G^{\prime}(\sigma(t) u)\right\| \\
& \leq-2 \delta \eta(\sigma(t) u) .
\end{aligned}
$$

Thus,

$$
\begin{gathered}
G(\sigma(t) u) \leq G(u), \quad t \geq 0, \\
G(\sigma(t) u) \leq a_{0}, \quad u \in A, t \geq 0, \\
\sigma(t) u=u, \quad u \in A, t \geq 0 .
\end{gathered}
$$

Again, this follows from the fact that

$$
G(\sigma(t) u) \leq a_{0} \leq b_{0}<a-3 \delta, \quad u \in A, t \geq 0 .
$$

Hence, $\eta(\sigma(t) u)=0$ for $u \in A, t \geq 0$. This means that

$$
\begin{gathered}
\sigma^{\prime}(t) u=0, \quad u \in A, t \geq 0, \\
\sigma(t) u=\sigma(0) u=u, \quad u \in A, t \geq 0 .
\end{gathered}
$$


Let

$$
E_{\alpha}=\{u \in E: G(u) \leq \alpha\}
$$

We note that there is a $T>0$ such that

$$
\sigma(T) E_{a+\delta} \subset E_{a-\delta}
$$

(In fact, we can take $T=1$.) Let $u$ be any element in $E_{a+\delta}$. If there is a $t_{1} \in[0, T]$ such that $\sigma\left(t_{1}\right) u \notin Q_{1}$, then

$$
G(\sigma(T) u) \leq G\left(\sigma\left(t_{1}\right) u\right)<a-\delta
$$

by (3.8). Hence $\sigma(T) u \in E_{a-\delta}$. On the other hand, if $\sigma(t) u \in Q_{1}$ for all $t \in[0, T]$, then $\eta(\sigma(t) u)=1$ for all $t$, and (3.8) yields

$$
G(\sigma(T) u) \leq G(u)-2 \delta T \leq a-\delta .
$$

Hence (3.13) holds. Now by (1.10), there is a $K \in \mathscr{K}_{U}(A)$ such that

$$
K \subset E_{a+\delta}
$$

Note that $\sigma(T) \in \Lambda_{U}(A)$. Let $\tilde{K}=\sigma(T)(K)$. Then $\tilde{K} \in \mathscr{K}_{U}(A)$ by Lemma 3.2. But

$$
\sup _{\widetilde{K}} G=\sup _{u \in K} G(\sigma(T) u)<a-\delta,
$$

which contradicts (1.10), proving the theorem for the case $b_{0}<a$.

Now assume that $b_{0}=a$. If there did not exist a sequence satisfying both (1.11) and (1.12), then there would be positive numbers $\epsilon, \delta, T$ such that $\delta<\epsilon T$ and (2.12) holds whenever

$$
u \in Q=\{u \in E: d(u, B) \leq 4 T,|G(u)-a| \leq 3 \delta\} .
$$

Let

$$
\begin{aligned}
& Q_{0}=\{u \in E: d(u, B) \leq 3 T,|G(u)-a| \leq 2 \delta\}, \\
& Q_{1}=\{u \in E: d(u, B) \leq 2 T,|G(u)-a| \leq \delta\} .
\end{aligned}
$$

Since $a=b_{0}$, we see that $Q_{1} \neq \phi$. Define $Q_{2}$ and $\eta(u)$ as before and let $\sigma(t)$ be the flow generated by the mapping (3.7) with everything now with respect to the new sets $Q_{j}$. Let $u$ be any element in $E_{a+\delta}$. If there is a $t_{1} \leq T$ such that $\sigma\left(t_{1}\right) u \notin Q_{1}$, then either

$$
G\left(\sigma\left(t_{1}\right) u\right)<a-\delta
$$

or

$$
d\left(\sigma\left(t_{1}\right) u, B\right)>2 T \text {. }
$$


Since

$$
\left\|\sigma(t) u-\sigma\left(t^{\prime}\right) u\right\| \leq\left|t-t^{\prime}\right|
$$

(3.21) implies that

$$
d(\sigma(t) u, B)>T, \quad 0 \leq t \leq T .
$$

On the other hand, if $\sigma(t) u \in Q_{1}$ for all $t \in[0, T]$, then

$$
G(\sigma(T) u) \leq G(u)-2 \epsilon T \leq a+\delta-2 \delta=a-\delta .
$$

Thus we have either

$$
G(\sigma(T) u)<a-\delta
$$

or (3.23) holds. Since $b_{0}=a$, this shows that

$$
\sigma(T) E_{a+\delta} \cap B=\phi .
$$

We also note that

$$
\sigma(t) A \cap B=\phi, \quad 0 \leq t \leq T .
$$

We have by (3.8) that

$$
G(\sigma(t) u) \leq a_{0}-2 \epsilon \int_{0}^{t} \eta(\sigma(\tau) u) d \tau, \quad u \in A .
$$

If $\sigma(t) u \in B$, we must have $G(\sigma(t) u) \geq b_{0} \geq a_{0}$. The only way this can happen is when

$$
\eta(\sigma(\tau) u) \equiv 0, \quad 0 \leq \tau \leq t .
$$

But this implies $\sigma(\tau) u \in \bar{Q}_{2}$ for such $\tau$, and this in turn implies either

$$
G(\sigma(\tau) u)<a-\delta, \quad 0 \leq \tau \leq t
$$

or

$$
d(\sigma(\tau) u, B)>2 T, \quad 0 \leq \tau \leq t .
$$

In either case, we cannot have $\sigma(t) u \in B$. Thus (3.27) holds. Let $K \in \mathcal{K}_{U}(A)$ satisfy (3.16), and let $\tilde{K}=\sigma(T) K$. Then $\tilde{K} \in \mathscr{K}_{U}(A)$. But (3.26) and (3.27) imply that $\tilde{K} \cap B=\phi$, contradicting the fact that $A$ is chained to $B$. This completes the proof of the theorem.

Now we give the proof of Theorem 1.9.

Proof. Assume that $\varphi \in \Lambda_{U}$ does not satisfy (1.6), and let $G(u)=\|\varphi(u)\|^{2}$. Then by the definition of the class $\Lambda_{U}, G \in C_{U}^{1}(E, \mathbb{R})$, sup $G(A)<\inf G(B)$, and $G$ has no critical level 
$c \geq \inf _{x \in B} G(x)>0$. To show the latter, assume that there is a sequence $u_{k}$ satisfying (1.3). Then we have for any bounded sequence $v_{k} \in E$,

$$
\left(\varphi^{\prime}\left(u_{k}\right) v_{k}, \varphi\left(u_{k}\right)\right) \longrightarrow 0 .
$$

Let $v_{k}:=\left(\varphi^{\prime}\left(u_{k}\right)\right)^{-1}\left(\varphi\left(u_{k}\right)\right)$. Then $\left(\varphi^{\prime}\left(u_{k}\right) v_{k}, \varphi\left(u_{k}\right)\right)=G\left(u_{k}\right) \rightarrow c$. However, the sequence $v_{k}$ is bounded: $G\left(u_{k}\right) \rightarrow c$ implies that $\varphi\left(u_{k}\right)$ is bounded, which implies that $u_{k}$, and consequently that $\left(\varphi^{\prime}\left(u_{k}\right)\right)^{-1}$, and $v_{k}$ are also bounded. Hence, $G^{\prime}\left(u_{k}\right) \rightarrow 0$ implies $G\left(u_{k}\right) \rightarrow 0$, showing that $c=0$. Thus, $A$ does not link $B$.

\section{Equivalent linking conditions}

In this section, we relate our present definition of linking to the one of [11].

Let $A, B \subset E$ be disjoint sets and assume that $A$ is bounded. Let $\Phi$ be a set of all maps $\psi \in C_{U}([0,1] \times E ; E)$ such that $\psi(0, \cdot)=\mathrm{id}_{E}$ and that there exists a point $\xi \in E$ such that $\psi(t, \cdot)$ converges to $\xi$ as $t \rightarrow 1$ uniformly on bounded sets. Let $\Psi \subset \Phi$. We will say that $A$ is $\Psi$-chained to $B$ if for every $\psi \in \Psi$,

$$
\psi(A,[0,1]) \cap B \neq \varnothing .
$$

Proposition 4.1. If $A$ is $\Phi$-chained to $B$, then $A$ is strongly chained to $B$.

Proof. Assume that $A$ is not strongly chained to $B$. Then there are a map $\varphi \in \Lambda$ and an $R>0$ such that $\varphi(A) \subset B_{R}(0)$ and $\varphi(B) \cap B_{R}(0)=\varnothing$. Let $\psi(t, u)=(1-t) u$. Then $\psi \in$ $\Phi$ and $[\psi(t, \cdot) \circ \varphi](A) \subset B_{R}(0)$. Therefore $[\psi(t, \cdot) \circ \varphi](A) \cap \varphi(B)=\varnothing$. This implies that $\left[\varphi^{-1} \circ \psi(t, \cdot) \circ \varphi\right](A) \cap B=\varnothing$. Since $\varphi^{-1} \circ \psi(t, \cdot) \circ \varphi \in \Phi$, this means that $A$ is not $\Phi$ chained to $B$, a contradiction.

Corollary 4.2. If $A$ is $\Phi$-chained to $B$, then $A$ links $B$.

Let

$$
\Phi_{0}:=\left\{\psi \in \Phi: \psi(t, \cdot) \in C_{U}^{1} \text { and } \forall t \in(0,1) \exists \varepsilon_{t}>0:\left\|\psi^{\prime}(t, \cdot)\right\| \geq \varepsilon_{t}\right\}
$$

Proposition 4.3. If $E$ is a Hilbert space and $A$ links $B$, then $A$ is $\Phi_{0}$-chained to $B$.

Proof. Assume that $A$ is not $\Phi_{0}$-chained to $B$. Then there are a map $\psi \in \Phi_{0}$ and a point $\xi \in E$ such that $\psi(t, \cdot) \rightarrow_{t \rightarrow 1} \xi$ and $\psi([0,1], A) \cap B=\varnothing$. Since $A$ is bounded, $\psi(t, \cdot)$ converges to $\xi$ uniformly on $A$. Then there are a $\tau \in(0,1)$ and an $r>0$ such that $\psi(\tau, A) \subset$ $B_{r}(\xi)$ and $B_{2 r}(\xi) \cap B=\varnothing$. Let $G(u)=\|\psi(\tau, u)-\xi\|$. The functional $G$ is in $C_{U}^{1}$ and it does not have a critical level $c>r$ since $\left\|\psi^{\prime}(\tau, \cdot)\right\|$ is bounded away from zero. Thus, $A$ does not link $B$.

Corollary 4.4. If $E$ is a Hilbert space, $A$ is strongly chained to $B, d(A, B)>0$, then $A$ is $\Phi_{0}$-chained to $B$.

\section{Acknowledgments}

The first author was supported in part by an NSF grant. The second author was supported by Veteskapsådet. 


\section{References}

[1] P. Bartolo, V. Benci, and D. Fortunato, Abstract critical point theorems and applications to some nonlinear problems with "strong" resonance at infinity, Nonlinear Analysis. Theory, Methods \& Applications 7 (1983), no. 9, 981-1012.

[2] V. Benci and P. H. Rabinowitz, Critical point theorems for indefinite functionals, Inventiones Mathematicae 52 (1979), no. 3, 241-273.

[3] H. Brezis and L. Nirenberg, Remarks on finding critical points, Communications on Pure and Applied Mathematics 44 (1991), no. 8-9, 939-963.

[4] K.-C. Chang, Infinite-Dimensional Morse Theory and Multiple Solution Problems, Progress in Nonlinear Differential Equations and Their Applications, 6, Birkhäuser Boston, Massachusetts, 1993.

[5] J. Mawhin and M. Willem, Critical Point Theory and Hamiltonian Systems, Applied Mathematical Sciences, vol. 74, Springer, New York, 1989.

[6] L. Nirenberg, Variational and topological methods in nonlinear problems, Bulletin of the American Mathematical Society. New Series 4 (1981), no. 3, 267-302.

[7] P. H. Rabinowitz, Minimax Methods in Critical Point Theory with Applications to Differential Equations, CBMS Regional Conference Series in Mathematics, vol. 65, American Mathematical Society, Rhode Island, 1986.

[8] M. Schechter, The intrinsic mountain pass, Pacific Journal of Mathematics 171 (1995), no. 2, 529-544.

[9] _ New linking theorems, Rendiconti del Seminario Matematico della Università di Padova 99 (1998), 255-269.

[10]_ Linking Methods in Critical Point Theory, Birkhäuser Boston, Massachusetts, 1999.

[11] M. Schechter and K. Tintarev, Pairs of critical points produced by linking subsets with applications to semilinear elliptic problems, Bulletin de la Société Mathématique de Belgique. Série B 44 (1992), no. 3, 249-261.

[12] Linking in Hilbert space, Proceedings of the American Mathematical Society 134 (2006), no. 2, 403-410.

[13] S. Z. Shi, Ekeland's variational principle and the mountain pass lemma, Acta Mathematica Sinica. New Series 1 (1985), no. 4, 348-355.

[14] E. A. de B. e. Silva, Linking theorems and applications to semilinear elliptic problems at resonance, Nonlinear Analysis. Theory, Methods \& Applications 16 (1991), no. 5, 455-477.

[15] K. Tintarev, Isotopic linking and critical points of functionals, Nonlinear Analysis. Theory, Methods \& Applications 30 (1997), no. 7, 4145-4149, Proceedings of the Second World Congress of Nonlinear Analysts, Part 7 (Athens, 1996).

[16] M. Willem, Minimax Theorems, Progress in Nonlinear Differential Equations and Their Applications, 24, Birkhäuser Boston, Massachusetts, 1996.

Martin Schechter: Department of Mathematics, University of California, Irvine,

CA 92697-3875, USA

E-mail address: mschecht@math.uci.edu

Kyril Tintarev: Department of Mathematics, Uppsala University, P.O. Box 480,

75106 Uppsala, Sweden

E-mail address: kyril.tintarev@math.uu.se 


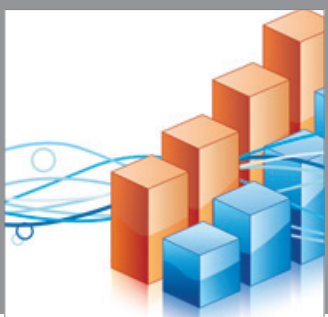

Advances in

Operations Research

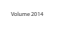

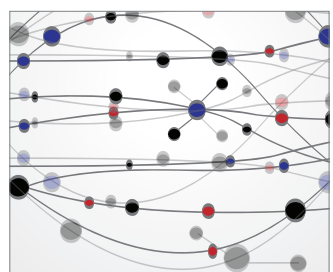

\section{The Scientific} World Journal
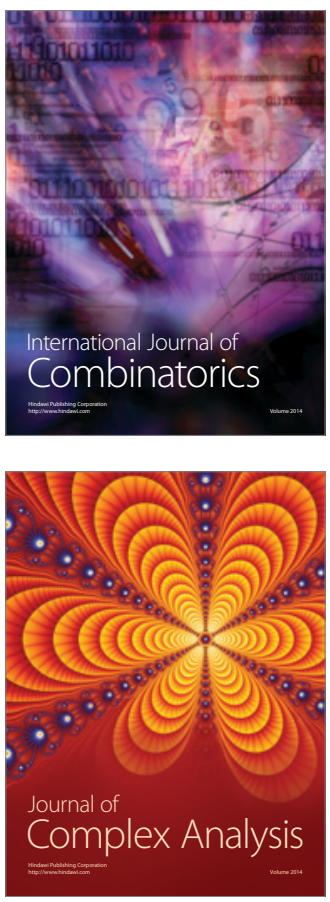

International Journal of

Mathematics and

Mathematical

Sciences
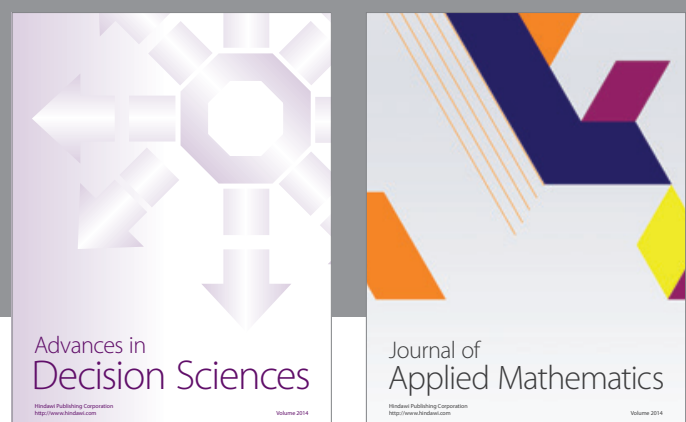

Journal of

Applied Mathematics
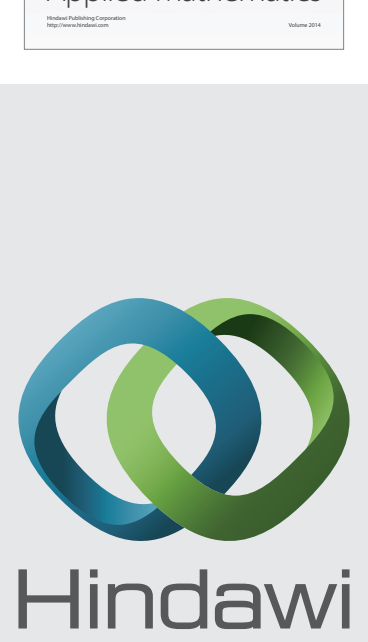

Submit your manuscripts at http://www.hindawi.com
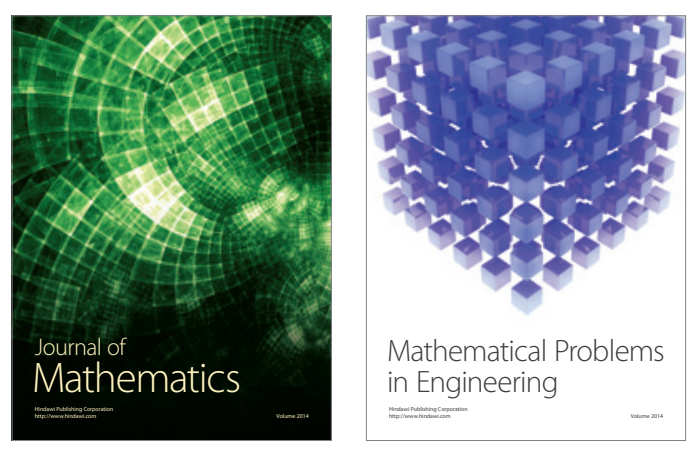

Mathematical Problems in Engineering
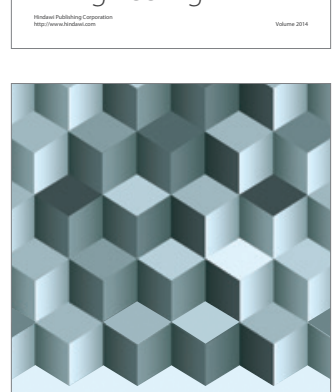

Journal of

Function Spaces
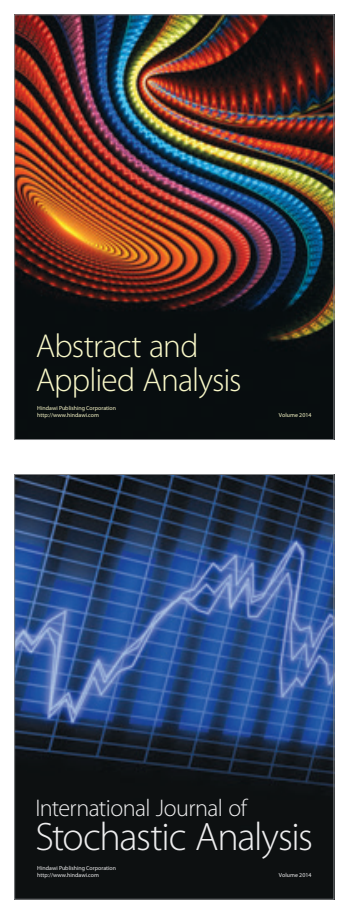

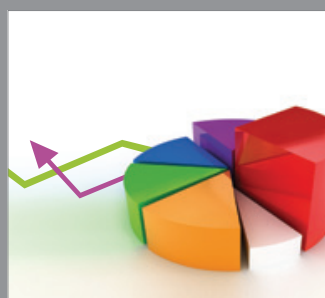

ournal of

Probability and Statistics

Promensencen
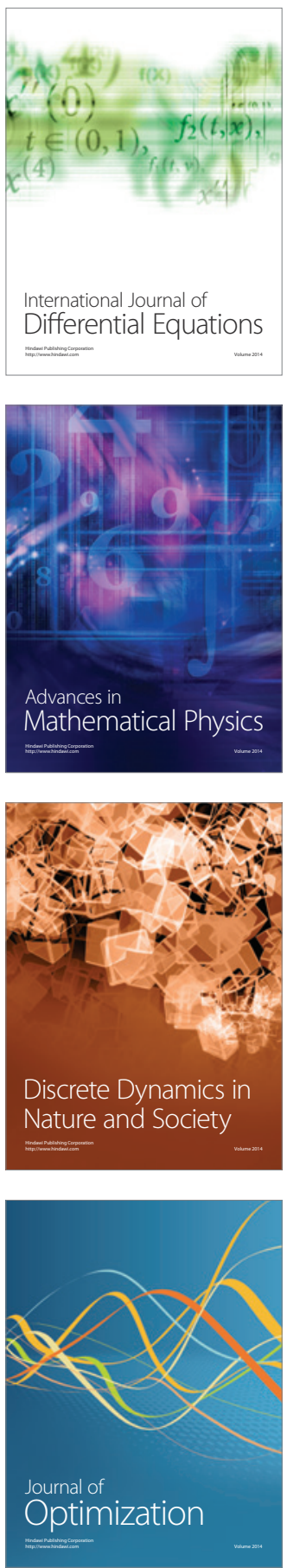